\title{
REVIEW
}

\section{Neurochemistry of Hypomyelination Investigated with MR Spectroscopy}

\author{
Jun-ichi TAKANASHI ${ }^{1,2 *}$ \\ ${ }^{1}$ Department of Pediatrics, Tokyo Women's Medical University, Yachiyo Medical Center \\ 477-96 Owadashinden, Yachiyo-shi, Chiba 276-8524, Japan \\ ${ }^{2}$ Molecular Imaging Center, National Institute of Radiological Sciences \\ (Received July 16, 2014; Accepted December 10, 2014; published online March 31, 2015)
}

Proton magnetic resonance spectroscopy (MRS) allows the noninvasive exploration of tissue metabolism in vivo, providing neurophysiological and neurochemical information. $\mathrm{N}$-acetylaspartate (NAA) is generally considered to be a marker of neurons and axons, and many neurodegenerative disorders, including demyelinating disorders, exhibit a decrease in total NAA (tNAA). MRS in human hypomyelination disorders, such as Pelizaeus-Merzbacher disease (PMD), is characterized by normal to elevated tNAA, elevated myo-inositol and creatine $(\mathrm{Cr})$, and normal to decreased choline (Cho).

MRS in the thalamus of a hypomyelinating mouse model, a myelin synthesis-deficient (msd) mouse, a model of connatal PMD with mutation of the Plp1 gene, revealed increased tNAA and $\mathrm{Cr}$ and decreased Cho. That of a shiverer mouse with an autosomal recessive mutation of the $M b p$ gene showed decreased Cho with normal tNAA and Cr. Accordingly, the reduction of Cho on MRS might be a common marker for hypomyelinating disorders. tNAA concentrations range from normal to increased, probably depending upon the underlying pathology of oligodendrocytes. tNAA may be increased in hypomyelination with a reduced number of mature oligodendrocytes, such as PMD.

\section{Keywords: hypomyelination, magnetic resonance spectroscopy, mouse model, myelin basic protein, proteolipid protein}

\section{Introduction}

Magnetic resonance (MR) imaging has greater sensitivity than computed tomography (CT) in the detection of lesions of the white matter that result from abnormal conditions of the myelin, including demyelination, hypomyelination, white matter rarefaction, and cystic degeneration. However, its specificity is limited because all these conditions result in $T_{2}$ prolongation of the white matter.

Proton MR spectroscopy (MRS) allows the noninvasive exploration of tissue metabolism in vivo, providing neurophysiological and neurochemical information. As the $N$-acetylaspartate (NAA) is synthesized in neuronal mitochondria and transported to axons (Fig. 1), ${ }^{1,2}$ NAA at $2.01 \mathrm{ppm}$ on MRS is generally considered an important marker of viable, functioning neurons and axons. NAA is ei-

\footnotetext{
${ }^{*}$ Corresponding author, Phone: +81-47-450-6000, E-mail: jtaka@twmu.ac.jp
}

ther released from the neuron or transported to oligodendrocytes, where it is catabolized by aspartoacylase (ASPA) into acetate and aspartate. NAA is also the precursor for the synthesis of $N$-acetylaspartylglutamate (NAAG) (2.04 ppm on MRS) in neurons. ${ }^{1,2}$ The biosynthesis or regulatory mechanisms of NAAG are poorly understood, but it modulates glutamatergic synaptic transmission and is released along with other neurotransmitters from neuronal synapses. Extracellular NAAG is hydrolyzed into NAA and glutamate by glutamate carboxypeptidase II (GCPII) on the membrane surface of astrocytes, and the breakdown products are taken in by the astrocytes. ${ }^{1,2}$ Accordingly, NAA is purported to have signal functions for axon-oligodendrocytes and NAAG, for axon-astrocytes.

The choline (Cho) peak at $3.2 \mathrm{ppm}$ likely contains various cell membrane precursors or breakdown products, such as phosphocholine, glycerophosphocholine, and phosphatidylcholine. Therefore, its elevation is seen in conditions of enhanced 


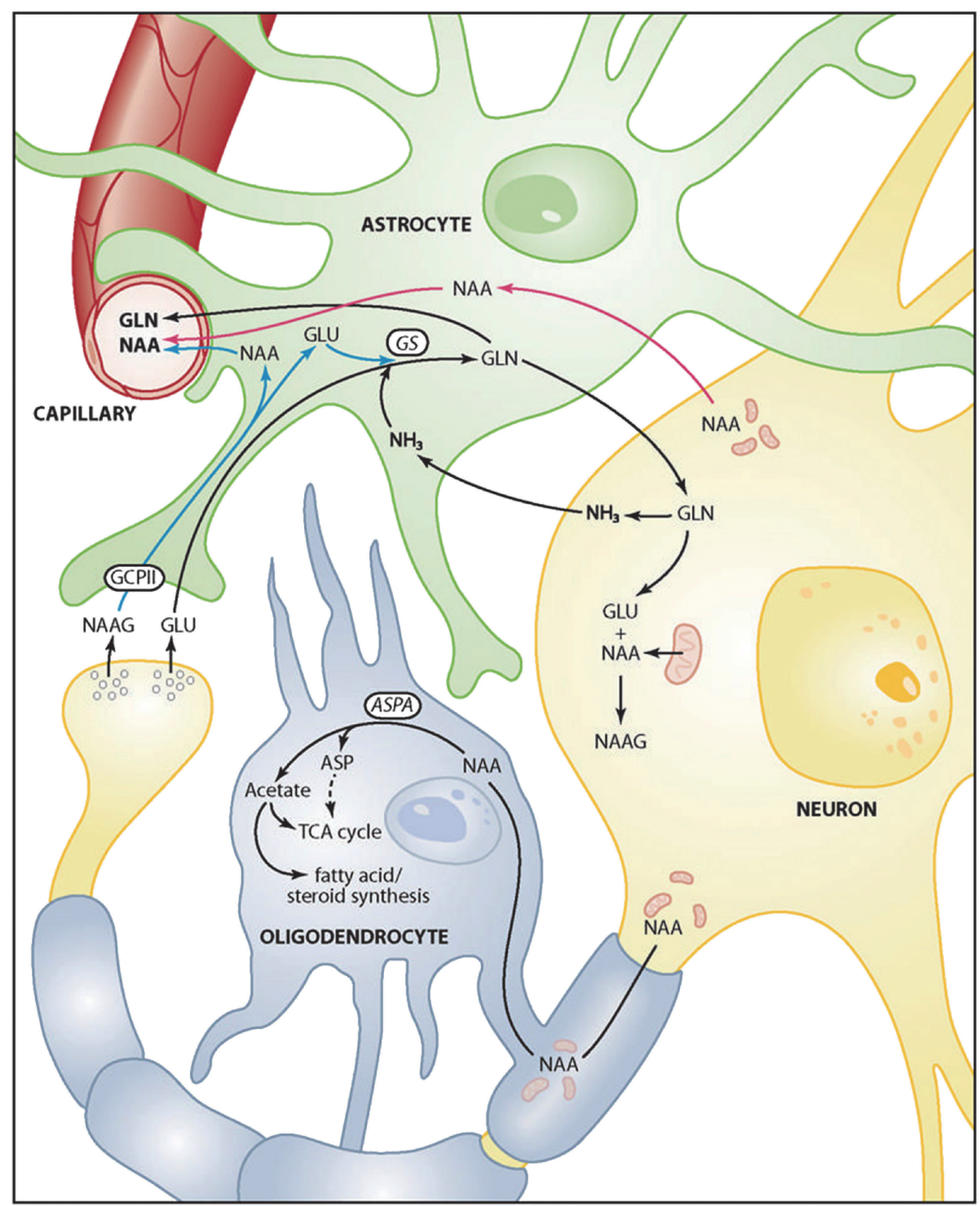

Fig. 1. Hypothetical model of $N$-acetylaspartate (NAA) synthesis and metabolism in the brain. NAA is synthesized in neuronal mitochondria and can either be transported to oligodendrocytes for fatty acid synthesis and energy production or used for the synthesis of $\mathrm{N}$-acetylaspartylglutamate (NAAG) in neurons. (Reprinted from Reference 2 with permission)

membrane turnover, such as myelination, active demyelination, and tumor growth. ${ }^{3,4}$ Thus, many neurodegenerative disorders, including demyelinating disorders (with increased Cho) and neuronal degenerative disorders, exhibit a decrease of total NAA (tNAA; NAA and NAAG, which are difficult to distinguish on clinical MRS with a 1.5- or 3-T magnet). ${ }^{5,6}$

I herein review MRS findings in hypomyelinating disorders of humans and their mouse models.

\section{Myelin Structure}

The myelin is a spiral membranous structure around the axons that comprises a protein-lipidprotein-lipid-protein structure. ${ }^{7,8}$ The lipid layers are composed of a bimolecular layer of hydrocarbon chains, cholesterol, phospholipids, and glycolipids. A dark line in electron microscopy, called the major dense line, contains myelin basic protein (MBP), an intracellular protein attached to the inner surface of the cell membrane and situated mainly in the cytoplasm (Fig. 2). A less electron-dense protein line, the intraperiod line, represents proteolipid protein (PLP) in the outer portion of the cell membrane and in the extracellular space (Fig. 2). The 2 major structural proteins of myelin, PLP and MBP, constitute 50\% (PLP) and 30\% (MBP) by weight of myelin proteins. The deficiency of PLP 
can cause hypomyelinating disorders, i.e., Pelizaeus-Merzbacher disease (PMD) in human and myelin synthesis-deficient (msd) mouse; and that of MBP can cause shiverer mouse.

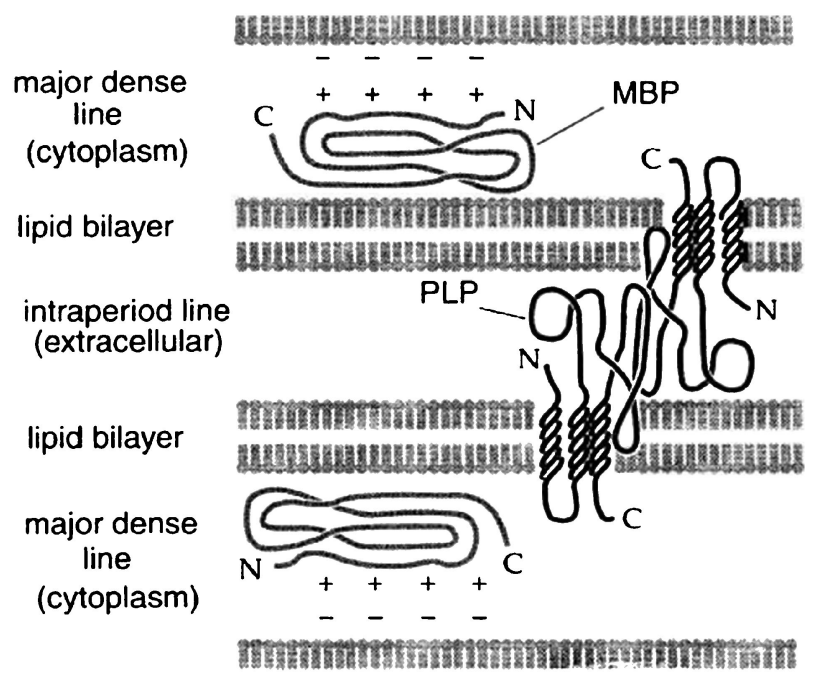

Fig. 2. Schematic of the structure of myelin. (Reprinted from Reference 7 with permission)

\section{MRS in Hypomyelinating Disorders in $\mathrm{Hu}-$ mans}

The term hypomyelination describes a permanent, substantial deficit of myelin deposition in the brain. In patients with classic-type PMD with duplication of the PLP1 gene, a representative hypomyelinating disorder, analysis of quantitative MRS with LCModel revealed increased tNAA, creatine $(\mathrm{Cr})$, and myo-inositol (mIns) with normal to decreased Cho (Fig. 3). ${ }^{9,10} \mathrm{mIns}$ is almost exclusively located in astrocytes and is recognized as an astrocyte-specific marker. ${ }^{11}$ Astrocytes also contain relatively high $\mathrm{Cr}$, compared with neurons. ${ }^{12}$ Concomitant astrogliosis observed in $\mathrm{PMD}^{13,14}$ probably results in increased mIns and Cr. It is not certain whether Cho is normal or decreased, but a reasonable decrease in Cho reflects decreased myelin synthesis and turnover, as observed in the brain in PMD. ${ }^{13,14} \mathrm{An}$ increased concentration of $\mathrm{Cr}$ prevents detection of increased tNAA when the tNAA/ $\mathrm{Cr}$ ratio is utilized. This may explain the failure in previous studies that utilized relative values to detect increased tNAA. ${ }^{15,16}$

A
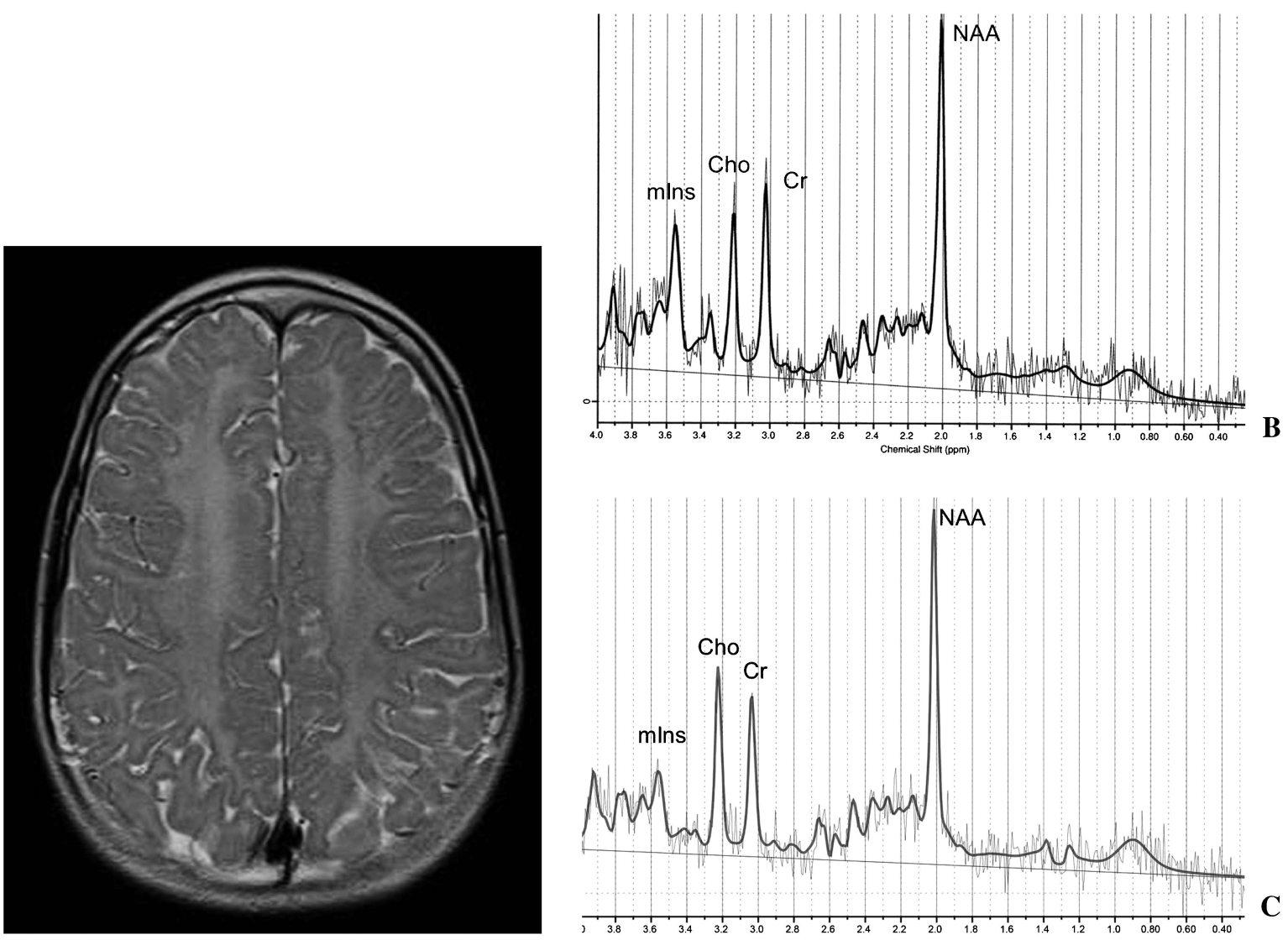

Fig. 3. Magnetic resonance (MR) imaging (A) and spectroscopy (MRS) (B) of a 12-year-old patient with Pelizaeus-Merzbacher disease ( $P L P 1$ duplication) shows $\mathrm{T}_{2}$ prolongation in the white matter; increased total $N$-acetylaspartate (tNAA), creatine (Cr), and myo-inositol (mIns); and reduced choline (Cho) (1.01 mM, normal mean \pm standard deviation [SD] $1.31 \pm 0.09 \mathrm{mM})$, as analyzed with LCModel. Normal MRS (C) of the 12-year-old boy showed a Cho concentration of $1.31 \mathrm{mM}$. 
In another hypomyelinating disorder, hypomyelination with atrophy of the basal ganglia and cerebellum (HABC), MRS showed normal tNAA and Cho with increased mIns and $\mathrm{Cr}^{17}{ }^{17}$ MRS in patients with Pelizaeus-Merzbacher-like disease (PMLD) also showed normal ratios of tNAA/Cr and Cho/ Cr. ${ }^{18}$ Based on these data, MRS in hypomyelination disorders in humans is characterized by elevated to normal tNAA, elevated mIns and $\mathrm{Cr}$, and normal to decreased Cho, findings distinct from those of MRS in active demyelination, which include decreased tNAA and increased Cho. ${ }^{6}$ The increase of tNAA may be explained by the higher axonal density in the absence of oligodendrocytes and myelin sheath $^{10}$; however, the exact mechanism is uncertain. Neither is it clear whether changes in the level of NAA or NAAG, or both, result in the increased tNAA.

\section{MRS in the Hypomyelinating Mouse Model}

I herein review findings of MRS studies and immunohistochemical analysis in 2 hypomyelinating mouse models to evaluate neurochemical derangement in hypomyelination.

\section{Mouse model and MRS methods}

The 2 mouse models of hypomyelination were the msd mouse, a model of connatal PMD with mutation of the Plp1 gene, and the shiverer mouse with an autosomal recessive mutation of the $M b p$ gene. ${ }^{19,20}$ Both models were subjected to MR imaging and MRS performed on a 7T MR imaging scanner. $T_{2}$ prolongation in the white matter, thalamus, and cortex on $\mathrm{T}_{2}$-weighted images reflected hypomyelination. ${ }^{19,20}$ For single-voxel ${ }^{1} \mathrm{H}-\mathrm{MRS}$, we chose a region of interest (ROI) predominantly in the thalamus, with volumes of interest of $3.0 \times$ $3.0 \times 3.0 \mathrm{~mm}$, because the white matter of mice is too thin for ROI placement. We combined outer volume suppression with a point-resolved spectroscopy sequence for signal acquisition (repetition time [TR]/echo time [TE], 2,500/20 ms for $\mathrm{msd}$ mice and 4,000/20 for shiverer mice). ${ }^{1} \mathrm{H}$-MRS was quantitatively analyzed using the water scaling method of LCModel, which uses the unsuppressed water signal obtained from the same ROI as an internal reference for quantification (default water proton density $[\mathrm{PD}]$ of $35.88 \mathrm{M}$ and $\exp ^{(-\mathrm{TE} / \mathrm{T} 2)}=$ $0.7) .^{21,22}$ The concentration was corrected by the $\mathrm{PD}$ and $\mathrm{T}_{2}$ value of the ROI, as multiplied by $\mathrm{R}=\mathrm{PD}$ of $_{\mathrm{ROI}} / 35.88 \times \exp ^{(-20 / \mathrm{T} 2 \text { of ROI })} / 0.7$; however, we used the default PD because we did not measure the PD of the ROI. ${ }^{19-22}$

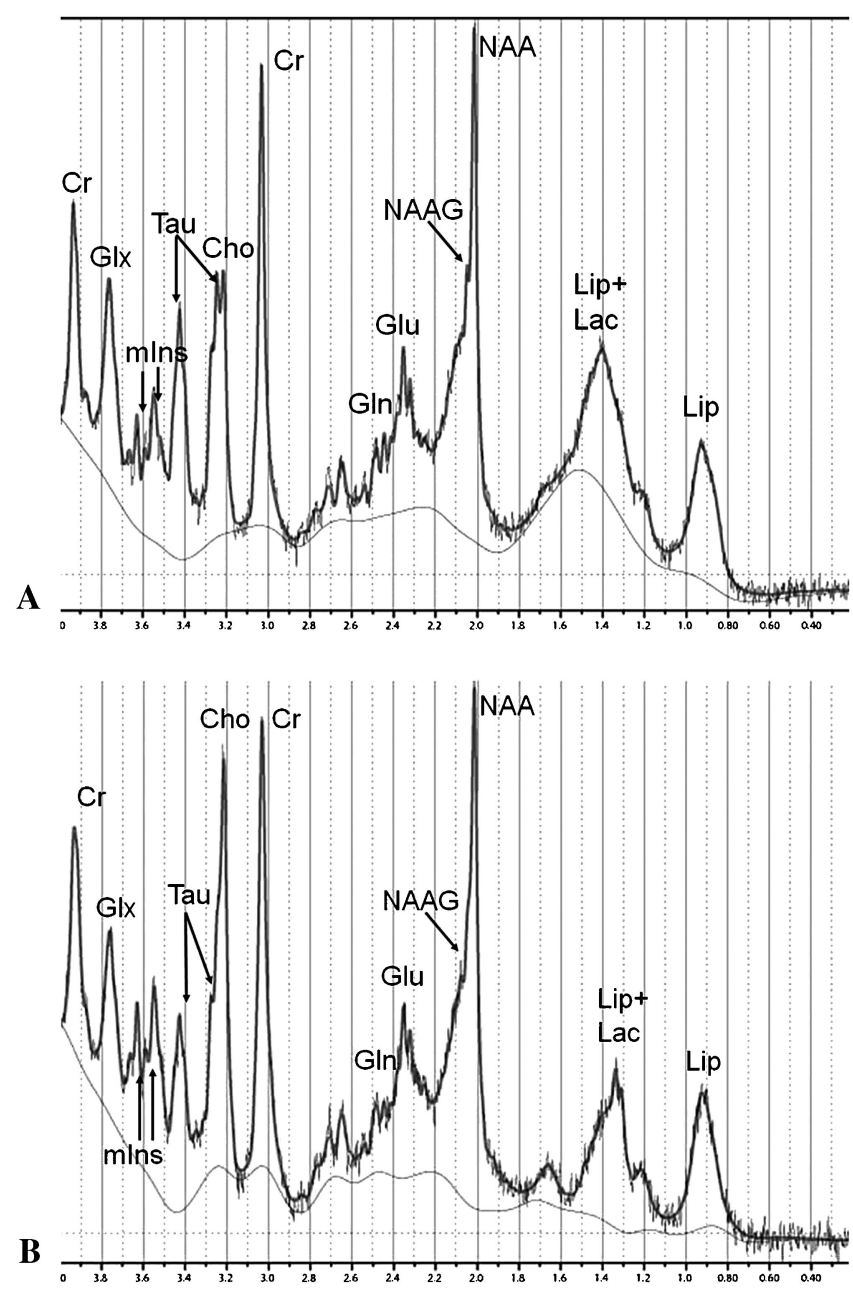

Fig. 4. Magnetic resonance spectroscopy (MRS) of an msd mouse (A) shows markedly reduced choline (Cho) compared with that in a wild-type mouse (B). (Reprinted from Reference 19 with permission)

\section{Brain metabolites measured by MRS}

Msd mouse. tNAA, Cr, glutamate, and glutamine were elevated, and Cho was decreased in msd mice $(-21.8 \%)$ compared with wild-type mice (Fig. 4). There was no difference in mIns. NAAG was not measured because LCModel did not permit acceptable reliability for NAAG. ${ }^{19}$

Shiverer mouse. Cho was decreased in the shiverer mice compared with heterozygous and wildtype mice. The degree of Cho reduction in the shiverer mice compared with that in wild-type mice $(-6.6 \%)$ was much less than that in the msd mice. Other metabolites did not differ among the 3 groups. We did not measure NAAG for of the same reason as in the msd mice. ${ }^{20}$

High performance liquid chromatography (HPLC) measurement of NAA and NAAG in msd mice

Levels of both NAA and NAAG were higher in the thalamus of msd mice (NAA, $3.52 \pm 0.37$; 
NAAG, $0.66 \pm 0.06 \mu \mathrm{mol} / \mathrm{g}$ tissue) than in wildtype mice (NAA, $2.90 \pm 0.43$; NAAG $0.57 \pm 0.07$ $\mu \mathrm{mol} / \mathrm{g}$ tissue $).{ }^{19}$

\section{Immunohistochemical Analysis}

Msd mouse. Immunostaining of Mbp, a marker for mature myelin sheath in msd mice, showed sparse and weak staining in the white matter, thalamus, and cortex compared with that in the wildtype mice. ${ }^{19}$ Immunostaining of glial fibrillary acidic protein (Gfap), a marker for astrocytes, of the msd mice (white matter and thalamus) showed dense and strong staining and increased positive cells compared with findings in the wild-type mice. These indicated hypomyelination and astrogliosis in the brain of the msd mice. Immunostaining of neuronal nuclear antigen (NeuN), a marker for neuronal cells, showed no obvious difference between the msd and wild-type mice.

Shiverer mouse. Luxol fast blue (LFB) staining and immunostaining of $\mathrm{Mbp}$ of shiverer mice revealed sparse and weak staining in the white matter, thalamus, and cortex compared with staining in heterozygous and wild-type mice, ${ }^{20}$ which indicated hypomyelination in the brains of the shiverer mice. Gfap immunostaining of the shiverer mice showed dense and strong staining in the white matter, which indicated astrogliosis, but no difference in the thalamus (ROI). NeuN immunostaining of shiverer mice revealed no obvious difference in the thalamus or white matter.

\section{Discussion}

MRS of the 2 mouse models of hypomyelination showed decreased Cho, which suggests a reduction of Cho as a common neurochemical derangement in hypomyelination. On the other hand, tNAA concentration differed between msd and shiverer mice. Increased tNAA (both NAA and NAAG) in the msd mice (model of PMD) is compatible with MRS in human PMD, ${ }^{13,14}$ whereas the concentration of tNAA is normal in shiverer mice. NeuN immunostaining of the thalamus in both msd and shiverer mice revealed no difference compared with that in wild-type mice; therefore, the number of neuronal cells in the thalamus seems unlikely to explain the difference in tNAA. These findings suggest that an increase in tNAA is not directly related to hypomyelination.

One possible explanation for the difference in tNAA is the different patterns of oligodendrocytes in shiverer and msd mice (Table). Mutant PLP1 proteins in msd mice are abnormally folded and ac-
Table. Different patterns of oligodendrocytes in shiverer and msd mice

\begin{tabular}{|c|c|c|c|c|c|}
\hline & $\begin{array}{l}\text { Oligodendrocyte } \\
\text { progenitor cell }\end{array}$ & $\rightarrow$ & $\begin{array}{c}\text { Mature } \\
\text { oligodendrocyte }\end{array}$ & $\rightarrow$ & $\begin{array}{l}\text { Myelin } \\
\text { sheath }\end{array}$ \\
\hline Shiverer & $\uparrow$ & $\rightarrow$ & $\uparrow$ & $\not \rightarrow$ & $\downarrow$ \\
\hline Msd & $\uparrow$ & $\not \rightarrow$ & $\downarrow$ & $\rightarrow$ & $\downarrow$ \\
\hline
\end{tabular}

cumulated in the endoplasmic reticulum, which results in the activation of an unfolded protein response that finally leads to apoptotic death of oligodendrocytes before normal myelination occurs. ${ }^{23}$ In msd and in jimpy, another mouse model of PMD, massive apoptosis of oligodendrocytes appears to induce proliferation of oligodendrocyte progenitor cells (OPC) in the white matter, ${ }^{24,25}$ which leads to an increase in OPCs and the absence of mature oligodendrocytes (Table). On the other hand, the absence of MBP protein in shiverer mice results in the failure of oligodendrocytes to form a compact myelin sheath. OPCs in shiverer mice, unlike those in msd mice, can differentiate into oligodendrocytes, and both OPCs and mature oligodendrocytes are increased in number by as much as 2 times (Table). ${ }^{26}$

NAA is one of the most abundant free amino acids in the brain (about 10mM in human) and generally considered an important marker of neurons and axons. ${ }^{1,2}$ NAA is either released from neurons or transported to oligodendrocytes, where it is catabolized by ASPA into acetate and aspartate, ${ }^{27}$ which are used for fatty acid and steroid synthesis, and energy production (Fig. 1). In msd mice, the absence or dysfunction of mature oligodendrocytes may either disable neuron-to-oligodendrocyte NAA transport or affect NAA catabolism in oligodendrocytes, which leads to accumulation of NAA in neurons. An elevated concentration of NAA also increases NAAG biosynthesis, ${ }^{2}$ which probably results in increased tNAA on MRS, as observed on MRS in patients with PMD. ${ }^{13,14}$ On the other hand, NAA in shiverer mice may be normally transported from neurons to oligodendrocytes and then catabolized into acetate and aspartate, leading to normal tNAA.

Cho reduction is much less in shiverer than msd mice, which may also be explained by the different patterns of oligodendrocytes. MRS in vitro demonstrated higher concentrations of Cho in cultured oligodendrocytes per se than neurons, astrocytes, and OPCs. ${ }^{12}$ The pathological difference in oligodendrocytes may explain the differences in Cho reduction in the 2 strains of hypomyelination, that is, the increased number of oligodendrocytes with se- 
vere hypomyelination in shiverer mice, ${ }^{26}$ which leads to a mild reduction of Cho, and the absence of oligodendrocytes with severe hypomyelination in msd mice, ${ }^{24}$ which results in a more severe reduction of Cho.

MRS showed a normal concentration of $\mathrm{Cr}$ in the thalamus of shiverer mice, though increased $\mathrm{Cr}$ has been observed in msd mice and human patients with PMD. ${ }^{9,10,19}$ Because the elevated $\mathrm{Cr}$ was considered to result from the increased number of astrocytes in human PMD, the difference may result from thalamic astrogliosis, which is present in $\mathrm{msd}^{19}$ and absent in shiverer ${ }^{20}$ mice. Astrogliosis was recognized in the white matter of shiverer mice, therefore, MRS in the white matter of shiverer mice may show increased Cr. Because mIns is almost exclusively located in astrocytes (astrocyte marker), its increase would also be expected in msd mice, as observed in patients with PMD. ${ }^{9,10}$ Nevertheless, mIns was normal in msd mice compared with wild-type mice. The exact mechanism is uncertain, but markedly decreased mIns and elevated glutamine are observed in patients with hepatic encephalopathy and disorders of the urea cycle. ${ }^{28}$ This has been explained by volume-regulatory mIns release in response to ammonia-induced astrocyte glutamine accumulation. Thus, it is possible that the lack of increase of mIns observed in msd mice may be a consequence of the elevated concentration of glutamine.

\section{Conclusions}

The reduction of Cho on MRS might be a common marker for hypomyelinating disorders. Ranges of tNAA from normal to increased probably depend upon the underlying pathology of oligodendrocytes. In hypomyelination, tNAA is increased with reduced numbers of mature oligodendrocytes, such as PMD.

\section{Acknowledgements}

The author would like to thank Ichio Aoki, Ph.D., Nobuhiro Nitta, M.E., and Ms. Sayaka Shibata (Molecular Imaging Center, National Institute of Radiological Sciences, Chiba, Japan) for their technical support, and Ken Inoue, M.D., Ph.D. (Department of Mental Retardation and Birth Defect Research, National Center of Neurology and Psychiatry, Kodaira, Japan) and Prof. Nobuaki Iwasaki (Department of Pediatrics, Ibaraki Prefectural University of Health Sciences, Amimachi, Japan) for providing mouse models. This study was supported in part by a grant-in-aid for Research on Measures for Intractable Diseases (H26-itaku (nan)-ippan023 ) and a grant for Research on Nervous and Mental Disorders (24-7), both from the Ministry of Health, Labor, and Welfare of Japan and a grantin-aid for Scientific Research (C-24591790) from the Japanese Society for Scientific Research.

\section{References}

1. Moffett JR, Namboodiri AM. Expression of N-acetylaspartate and $\mathrm{N}$-acetylaspartylglutamate in the nervous system. Adv Exp Med Biol 2006; 576:7-26.

2. Moffett JR, Ross B, Arun P, Madhavarao CN, Namboodiri AM. N-acetylaspartate in the CNS: from neurodiagnostics to neurobiology. Prog Neurobiol 2007; 81:89-131.

3. Grodd W, Krägeloh-Mann I, Klose U, Sauter R. Metabolic and destructive brain disorders in children: findings with localized proton MR spectroscopy. Radiology 1991; 181:173-181.

4. Howe FA, Maxwell RJ, Saunders DE, Brown MM, Griffiths JR. Proton spectroscopy in vivo. Magn Reson Q 1993; 9:31-59.

5. Cecil KM, Kos RS. Magnetic resonance spectroscopy and metabolic imaging in white matter diseases and pediatric disorders. Top Magn Reson Imaging 2006; 17:275-293.

6. van der Voorn JP, Pouwels PJ, Hart AA, et al. Childhood white matter disorders: quantitative MR imaging and spectroscopy. Radiology 2006; 241:510517.

7. Barkovich AJ. Concepts of myelin and myelination in neuroradiology. AJNR Am J Neuroradiol 2000; 21:1099-1109.

8. van der Knaap MS. Myelin and white matter, In: van der Knaap MS, Valk J, eds. Magnetic resonance of myelination and myelin disorders. 3rd ed. Berlin: Springer, 2005; 1-19.

9. Takanashi J, Inoue K, Tomita M, et al. Brain N-acetylaspartate is elevated in Pelizaeus-Merzbacher disease with PLP1 duplication. Neurology 2002; 58: 237-241.

10. Hanefeld FA, Brockmann K, Pouwels PJ, Wilken B, Frahm J, Dechent P. Quantitative proton MRS of Pelizaeus-Merzbacher disease: evidence of dys- and hypomyelination. Neurology 2005; 65:701-706.

11. Danielsen ER, Ross B. The clinical significance of metabolites, In: Danielsen ER, Ross B. eds. Magnetic resonance spectroscopy diagnosis of neurological diseases. New York: Marcel Dekker, 1999; 23-43.

12. Urenjak J, Williams SR, Gadian DG, Noble M. Proton nuclear magnetic resonance spectroscopy unambiguously identifies different neural cell types. J Neurosci 1993; 13:981-989.

13. Seitelberger F. Pelizaeus-Merzbacher disease, In: Vinken PJ, Bruyn GW, eds. Handbook of clinical neurology. Vol 10: Leukodystrophies and poliodystrophies. Amsterdam: North Holland, 1970; 150-202. 
14. Hudson LD. Pelizaeus-Merzbacher disease and the allelic disorder X-linked spastic paraplegia type 2, In: Scriver CR, Beaudet AL, Sly WS, Valle D, eds. The metabolic and molecular bases of inherited disease. 8th ed. New York: McGraw-Hill, 2001; 57895798.

15. Takanashi J, Sugita K, Osaka H, Ishii I, Niimi H. Proton MR spectroscopy in Pelizaeus-Merzbacher disease. AJNR Am J Neuroradiol 1997; 18:533-535.

16. Bonavita S, Schiffmann R, Moore DF, et al. Evidence for neuroaxonal injury in patients with proteolipid protein gene mutations. Neurology 2001; 56: 785-788.

17. van der Knaap MS, Naidu SB, Pouwels PJ, et al. New syndrome characterized by hypomyelination with atrophy of the basal ganglia and cerebellum. AJNR Am J Neuroradiol 2002; 23:1466-1474.

18. Bugiani M, Al Shahwan S, Lamantea E, et al. GJA12 mutations in children with recessive hypomyelinating leukoencephalopathy. Neurology 2006; 67:273279.

19. Takanashi J, Saito S, Aoki I, Barkovich AJ, Ito Y, Inoue $\mathrm{K}$. Increased $\mathrm{N}$-acetylaspartate in model mouse of Pelizaeus-Merzbacher disease. J Magn Reson Imaging 2012; 35:418-425.

20. Takanashi J, Nitta N, Iwasaki N, et al. Neurochemistry in shiverer mouse depicted on MR spectroscopy. J Magn Reson Imaging 2014; 39:1550-1557.
21. Takanashi J, Somazawa F, Maruyama K, Terada H, $\mathrm{Xu} \mathrm{D,} \mathrm{Barkovich} \mathrm{AJ.} \mathrm{Metabolic} \mathrm{changes} \mathrm{in} \mathrm{early}$ childhood using LCModel with corrected water scaling method. J Magn Reson Imaging 2012; 35:174180.

22. Provencher SW. LCModel \& LCMgui user's manual. http://s-provencher.com/pub/LCModel/manual/ manual.pdf.

23. Gow A, Southwood CM, Lazzarini RA. Disrupted proteolipid protein trafficking results in oligodendrocyte apoptosis in an animal model of PelizaeusMerzbacher disease. J Cell Biol 1998; 140:925-934.

24. Skoff RP. Increased proliferation of oligodendrocytes in the hypomyelinated mouse mutant-jimpy. Brain Res 1982; 248:19-31.

25. Nishiyama A. Glial progenitor cells in normal and pathological states. Keio J Med 1998; 47:205-208.

26. Bu J, Banki A, Wu Q, Nishiyama A. Increased NG2+ glial cell proliferation and oligodendrocyte generation in the hypomyelinating mutant shiverer. Glia 2004; 48:51-63.

27. Bhakoo KK, Craig TJ, Styles P. Developmental and regional distribution of aspartoacylase in rat brain tissue. J Neurochem 2001; 79:211-220.

28. Takanashi J, Kurihara A, Tomita M, et al. Distinctly abnormal brain metabolism in late-onset ornithine transcarbamylase deficiency. Neurology 2002; 59: 210-214. 